agreement in some, between optical and radio polarization directions could perhaps be explained partly by Faraday rotation, partly by the fact that optical absorbers and nonthermal radio emitters do not occur in the same regions of space.

The one important conclusion one can draw from the above is that the radio data do not seem to be representative of a general magnetic field. This then raises the question whether the galactic nonthermal background is really a. smooth background or consists of a large number of extended discrete sources. The few regions where the polarized intensity is large would then be the nearest ones of these sources. The sources could have their own magnetic field and perhaps also contain a concentration of relativistic electrons. In directions where the line of sight through the Galaxy is very long, the background radiation would be smooth. In regions like the anticentre, however, one might expect the smooth background to break up into individual pieces. This seems indeed to have been observed (R. D. Davies, private communication). Also, at least part of the so-called "galactic halo" radiation could well be due to nearby emitters; it shows a considerable amount of structure.

On the basis of present evidence, therefore, one should consider the possibility that the magnetic field is not a smooth field following the spiral arms, but perhaps a broken-up field. One is tempted to consider this field as a result of old supernova explosions, the optical remnants of which have long since disappeared, but of which the radio emission is still appreciable, either by stronger radiation from the smoothly distributed relativistic electrons, or by radiation from its own electrons. Such a model would be consistent with the optical and radio observations of polarization, and with the observations of the continuous background emission.

\title{
References
}

BeHr, A. (1959).-Nachr. d. Akad. d. Wiss. Göttingen, math.-phys. Klasse Nr 7: 185-240.

Brouw, W. N., and Berkhuysen, E. M. (1962).-Nature 196: 757-78.

Brouw, W. N., Muller, C. A., and Tinbergen, J. (1962).-B.A.N. 16: 213-24.

HALL, J. S. (1958).-Pub. U.S. Naval Obs. 17 : 273-342.

Muller, C. A., Berkhuysen, E. M., Brouw, W. N., and Tinbergen, J. (1963).-Nature 200: 155-6.

Westerhout, G., Seeger, C. L., Brouw, W. N., and Tinbergen, J. (1962).-B.A.N. $16: 187-212$.

Wielebinski, R., Shakeshaft, J. R., and Pauliny-Toth, I. I. K. (1962).-Observatory 82 : 158-64.

Wielebinski, R., and Shakeshaft, J. R. (1962).-Nature 195: 982-3.

\section{RADIO POLARIZATION EFFECTS IN THE GALAXY}

\section{F. F. GARDNer}

\section{CSIRO Radiophysics Laboratory, Sydney}

Polarization effects involving our Galaxy have been studied in two ways : firstly, by observations of the polarization of the galactic radiation itself, and secondly, by the effects of galactic magnetic fields and ionization on the polarization of extragalactic radio sources. For an aerial of fixed dimensions, the intensity of the background varies at about $\lambda^{2 \cdot 5}$ ( $\lambda$ is wavelength) and that of the sources at about $\lambda^{0 \cdot 7}$. Thus background measurements are restricted to the longer wavelengths where the 
radiation is sufficiently intense, while sources are most easily studied at the shorter wavelengths where background confusion is small. The background radiation includes a component from small unresolved extragalactic radio sources in addition to the galactic radiation which is of interest in the first type of measurement. Both types of observation have been made with the 210 -foot aerial at Parkes; the observations of sources were done in collaboration with J. B. Whiteoak and that of the galactic radiation with J. A. Roberts, assisted by J. B. Whiteoak and A. G. Little for part of the time.

The measurement of the polarization of a radio source consists essentially of scanning across it with different aerial polarizations. For almost all the sources, the position angle of the electric field varies as $\lambda^{2}$ (Gardner and Whiteoak 1963) over the range of measurement, usually 10 to $30 \mathrm{~cm}$, a result which is consistent with Faraday rotation between the source and ourselves (Cooper and Price 1962). The relationship can be expressed (Gardner and Whiteoak 1963) as

$$
\theta=\theta_{\text {intr. }}+R_{M} \lambda^{2}
$$

where $\theta_{\text {intr. }}$ is the intrinsic polarization angle obtained by extrapolation to zero wavelength, and, on the hypothesis of synchrotron emission, is related to the magnetic

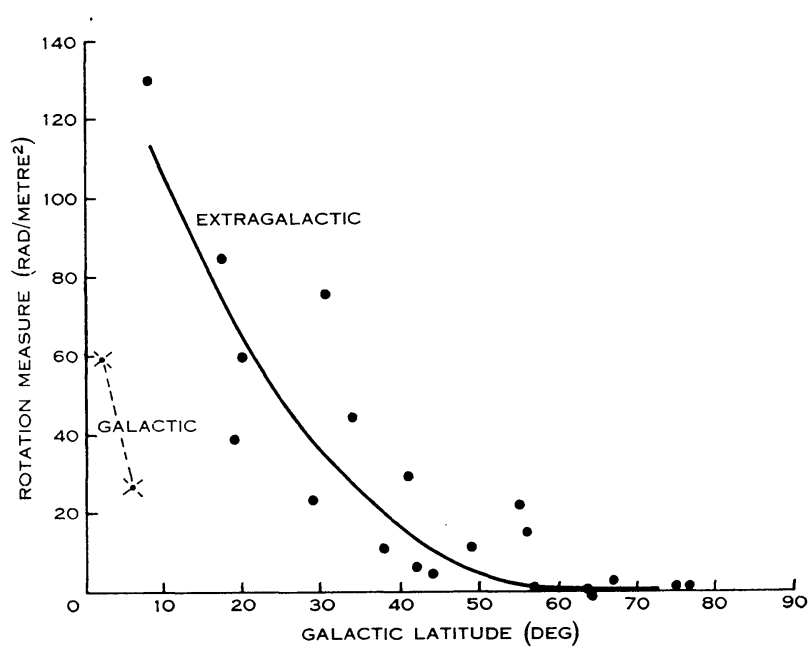

Fig. 1.-Absolute value of rotation measure against galactic latitude for extragalactic and galactic sources separately.

field direction in the source, while the rotation measure $\left(R_{\mathrm{M}}\right)$ measures the integrated value of the longitudinal field-electron density product along the line of sight. Numerically, with $R_{M}$ expressed in radians $/(\lambda \text { in metres })^{2}$,

$$
R_{\mathrm{M}}=8 \cdot 1 \int N H_{L} \mathrm{~d} L
$$

where electron density $N$ is in electrons per c.c., longitudinal magnetic field $H_{L}$ is in gamma $=10^{-5}$ gauss, and path $L$ is in parsecs.

In Figure 1 the absolute value of the rotation measure is plotted against galactic latitude. It is evident that for extragalactic sources the rotation measure is very small 
above latitude $60^{\circ}$ and increases appreciably towards the plane. This is fairly convincing evidence that the Faraday rotation for most radio sources is taking place within our own Galaxy.

In Figure 2 the positions of the radio sources measured are plotted in galactic coordinates, with the magnitude and sign of their rotation measures indicated approximately. A positive rotation measure corresponds to a magnetic field directly towards us. Apart from the general tendency for $R_{\mathrm{M}}$ to increase towards the plane, just noted, there does not appear to be a systematic pattern in the distribution which can be related to galactic structure. There is an obvious need for the measurement of more sources.

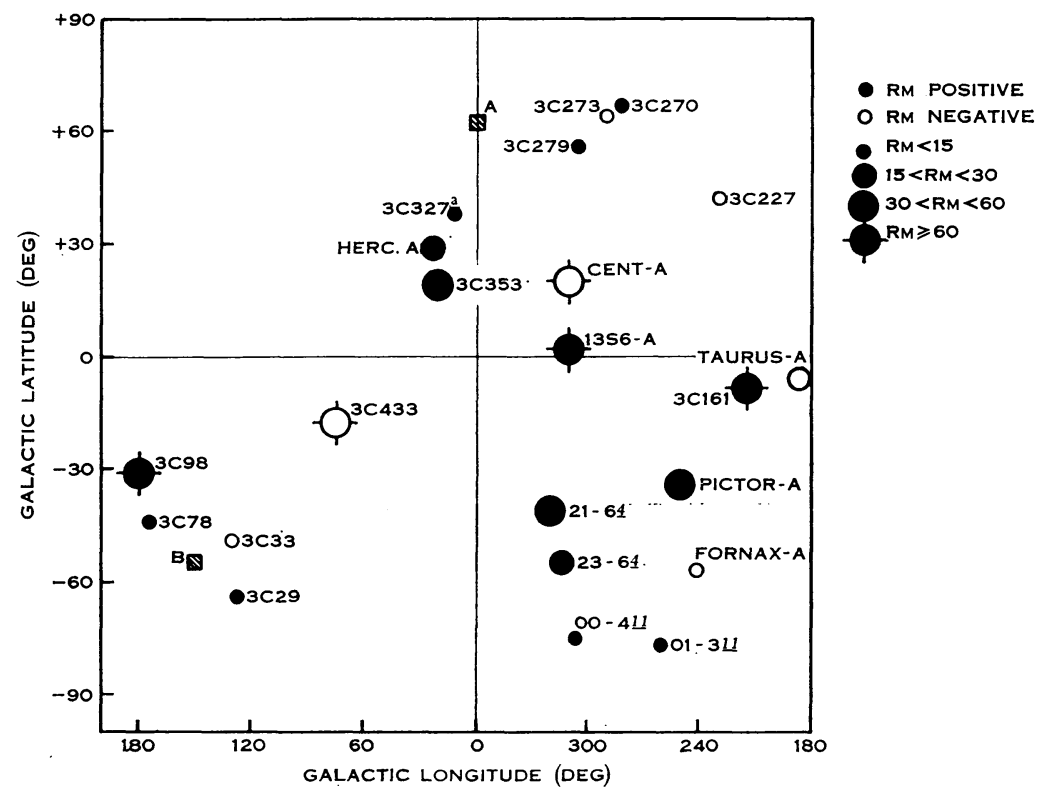

Fig. 2.-Galactic positions of sources measured. The magnitude of $R_{\mathrm{M}}$ is indicated by the dot size. Taurus $A$ and 13S6A are galactic sources, the remainder are extragalactic.

In general the degree of polarization decreases with increasing wavelength, but the way in which this depolarization takes place varies markedly from source to source. As pointed out by Gardner and Whiteoak (1963), a part of this depolarization might occur within the source, but there is an indication of galactic dependence. Further information is required before firm conclusions can be reached.

Linear polarization of the background radiation was first detected by Westerhout et al. (1962) using the 25-metre Dwingeloo paraboloid at a wavelength of $75 \mathrm{~cm}$. Their results are discussed in this volume (Paper 34) by Westerhout. Broadly, our objectives in the investigation of galactic polarization are the same as theirs. In addition, observations can be extended to the southern sky and, by using a larger aerial, the polarization can be studied in finer detail. It seems particularly important to measure the Faraday rotation associated with the radiation in order to determine the intrinsic polarization, related to the transverse magnetic field component at 
emission, and the rotation measure related to the longitudinal component. The determination may be complicated in practice if emission occurs over a volume in which Faraday rotation is also taking place. The other significant measurement is that of the spectrum of the polarization, as it may permit the determination of the energy spectrum of the relativistic electrons in a restricted region, selected on the basis of polarization.

Westerhout et al. found three regions where the polarization was particularly high. Two of these are accessible to us and are being investigated. They are in the vicinity of R.A. $14^{\mathrm{h}}, \delta+10^{\circ}\left(l^{\mathrm{II}} \approx 0^{\circ}, b^{\mathrm{II}} \approx+65^{\circ}\right)$, and R.A. $02^{\mathrm{h}}, \delta+10^{\circ}\left(l^{\mathrm{II}} \approx 150^{\circ}\right.$, $b^{\mathrm{II}} \approx-50^{\circ}$ ) and are marked $(\mathrm{A})$ and $(\mathrm{B})$ in Figure 2. The former is part of the so-called "northern spur", a ridge of emission coming out from the plane near $16^{\mathrm{h}}$ R.A., while the latter area is rather devoid of features apart from the polarization. Polarization has been found in a number of other parts of the sky, two of which are near $22^{\mathrm{h}}$, $-10^{\circ}$ and $01 \frac{1}{2}^{\mathrm{h}},-35^{\circ}$. In addition Mathewson, Milne, and Healey have made a survey around the Small Magellanic Cloud. Our results are preliminary and may be modified appreciably when the analysis is complete.

The measurements are complicated by the presence of a spurious polarization component which has to be subtracted from the observed values to give the true polarization at each point in the sky. The uncertainty in the estimation of this spurious component is at present the factor limiting the accuracy of polarization measurements. Westerhout et al. consider that the spurious component is constant and determined by aerial responses well outside the main beam. Our observations agree essentially with this interpretation, but have shown that the spurious component does vary with the zenith angle. With this spurious component allowed for, our values of polarization intensity agree reasonably with those of Westerhout et al. for regions (A) and (B). Differences in position angles are compatible with expected differences in Faraday rotation in the terrestrial ionosphere.

Multi-frequency observations from 375 to $450 \mathrm{Mc} / \mathrm{s}(67-80 \mathrm{~cm})$ have been made in both overlap regions. For region $(\mathrm{B})$ the rotation is small with an average of $11^{\circ}$ change from 375 to $450 \mathrm{Mc} / \mathrm{s}$ corresponding to $R_{\mathrm{M}}=+1 \mathrm{rad} / \mathrm{metre}^{2}$ if the relation with $\lambda^{2}$ is linear. This rotation would make the true polarization at emission $30^{\circ}$ less than the value measured at $75 \mathrm{~cm}$. For (A), there does seem to be a significant change from about $+1 \mathrm{rad} / \mathrm{metre}^{2}$ at R.A. $14^{\mathrm{h}} 08^{\mathrm{m}}, \delta+12^{\circ} 30^{\prime}$ to $+3 \mathrm{rad} / \mathrm{metre}^{2}$ at $14^{\mathrm{h}} 22^{\mathrm{m}},+14^{\circ} 15^{\prime}$. At the latter point the observed $75-\mathrm{cm}$ polarization would differ by about $90^{\circ}$ from the true value at emission. It may be significant that the rotation measure values are of the same order as for sources in the same regions of the sky. In both regions the sign of $R_{\mathrm{M}}$ is opposite to that of the ionosphere, which is negative and normally under $0.5 \mathrm{rad} / \mathrm{metre}^{2}$.

The measurement of the spectral slope of the polarized radiation component from observations over such a restricted range of wavelengths $(67-80 \mathrm{~cm})$ is very sensitive to errors in the estimation of the spurious polarization, and at present the spectral measurements are only significant for regions where the polarization is high (some $10^{\circ} \mathrm{K}$ peak-to-peak). It does appear that in region $(B)$ the polarized component has a very steep spectrum with an average around $\lambda^{4}$. In region (A) the spectrum is intermediate between that in (B) and a normal spectrum, $\lambda^{2 \cdot 5}$. 
Both types of polarization investigation are still in the preliminary stages, but promise to contribute significantly to our understanding of galactic emission and magnetic fields.

\title{
References
}

Cooper, B. F. C., and Price, R. M. (1962).-Nature 195: 1084-5.

GARDNER, F. F., and WHITEOAK, J. B. (1963).--Nature 197 : 1162-4.

Westerhout, G., Seeger, C. L., Brouw, W. N., and Tinbergen, J. (1962).-B.A.N.16: 187-212.

\section{Discussion}

Westfold: I think some of us would like to be assured by the observers that they are not taking linear polarization for granted. By doing so they are putting their faith in the synchrotron process of emission together with an isotropic distribution of electron velocities. It is desirable that, at least in selected areas, a number of measurements sufficient to determine all the Stokes parameters should be taken. If it were found that linear polarization was not always present it would indicate the need for an alternative hypothesis, or at least the relaxation of the assumption of isotropy. Again, if the polarization of the spurious spill-over contribution were not linear, this might offer a means of separating the spurious component from the main cosmic contribution in which we are interested.

Gardner: We have attempted to detect circular polarization in an extended region in Centaurus A which is approximately $50 \%$ linearly polarized, and also in Jupiter, $25 \%$ linearly polarized. In each case, we obtained less than $4 \%$ circular polarization.

Westerhout: The Dwingeloo observers are indeed aware of the fact that one should try elliptical polarization as well. They have not got that far yet.

The spurious signal comes from the ground only, and is a function of altitude, irrespective of what its origin is. Therefore it can be easily subtracted.

The scatter Dr. Gardner finds in his intensities is exactly of the same kind as we had in Dwingeloo during our first measurements. Improvements in feed and receiver made them disappear. During the daytime, considerable scatter remains owing to solar radiation in the side-lobes.

Lequeux: In connection with Dr. Westfold's remarks, I should like to say that Le Roux has shown that the polarization of synchrotron radiation of a single electron is not linear but elliptical with an ellipticity of the order of $\frac{1}{7}$, the amount of which depends on the isotropy of the velocities of the relativistic electrons and of their energy spectrum. This could perhaps be observed in radio sources.

\section{AN OUTLINE OF THE SPIRAL STRUCTURE OF THE SOUTHERN MILKY WAY}

\author{
B. J. BoK \\ Mount Stromlo Observatory
}

\section{Introduction}

It has become clear in recent years that the spiral features of our Galaxy like those of all galaxies - are of recent origin and are presumably short-lived phenomena. To trace them optically, we need to confine ourselves to concentrations in the interstellar gas and to stars and star groupings recently formed from these. We are hence limited primarily to $\mathrm{OB}$ associations and star clusters in which the earliest spectral types for the stars are not later than B2, preferably 05 to B1. It is most important that radial velocities be measured for a fair sampling of these stars, 